\title{
Un recorrido por nuestra experiencia en la inclusión de software dinámico en el diseño de materiales didácticos
}

\author{
José Luis Soto Munguía1, César Fabián Romero Félix ${ }^{2}$ \\ e-mail: 1joseluis.soto@unison.com, ${ }^{2}$ cesar.romero@unison.mx \\ Universidad de Sonora
}

\section{Resumen}

Se presentan aquí algunas de las experiencias en el uso de software de matemáticas dinámicas para el diseño de materiales y actividades didácticas. Partiendo del uso de Cabri fuera del aula, como las exploraciones gráficas de un profesor sobre temas avanzados para explorar los alcances del software, se generó toda una línea de trabajo en Matemática Educativa, pasando por la exploración novedosa de temas clásicos en matemáticas, al estudio de dificultades de aprendizaje y propuestas para su superación, culminando en una propuesta metodológica para el diseño de propuestas de enseñanza apoyadas en esta tecnología. Se ilustran algunos de los proyectos más representativos en esta línea de trabajo, así como algunas de las dificultades técnicas y didácticas que se han ido superando. A modo de conclusión, se presentan algunos ejemplos del proyecto que esta línea actualmente promueve.

Palabras clave: Software dinámico, Cabri, GeoGebra, diseño de materiales didácticos

Recibido 7 de febrero de 2021

Aceptado 14 de abril de 2021

\section{Cabri, los retos técnicos}

Desde que Cabri Geometry II apareció en el mercado a principios de los 90, provocó grandes expectativas sobre su impacto en la enseñanza de la Geometría, sobre todo en el nivel básico. Para muchos de quienes ejercemos la docencia en el nivel superior, representó una herramienta poderosa con la que podrían privilegiarse las representaciones gráficas dinámicas, que nos ofrecía la posibilidad de utilizar representaciones geométricas manipulables directamente en la pantalla de una computadora.

\subsection{La potencia de Cabri a prueba}

Para muchos profesores de matemáticas e investigadores en Matemática Educativa, interesados en esta nueva herramienta, el primer reto fue aprender a usarla. Surgieron así algunos grupos en el país, interesados en explorar la potencia de Cabri sin un propósito didáctico claro, pero que intentaban construir applets suficientemente sofisticados para mostrar el potencial del software. Estas construcciones no estaban enmarcadas en ningún proyecto particular y terminaban presentándose como "demos" en algún evento académico, pero permitieron especializar a una camada de usuarios de Cabri en el manejo de este software. De esos tiempos proviene la construcción que se muestra en la Figura 1, en la cual se simula una pecera con un pez que nadaba en su interior, mientras la pecera se vaciaba. Si se toma en cuenta que todavía no existía Cabri 3D, se entenderá que se trata de una construcción muy complicada. 

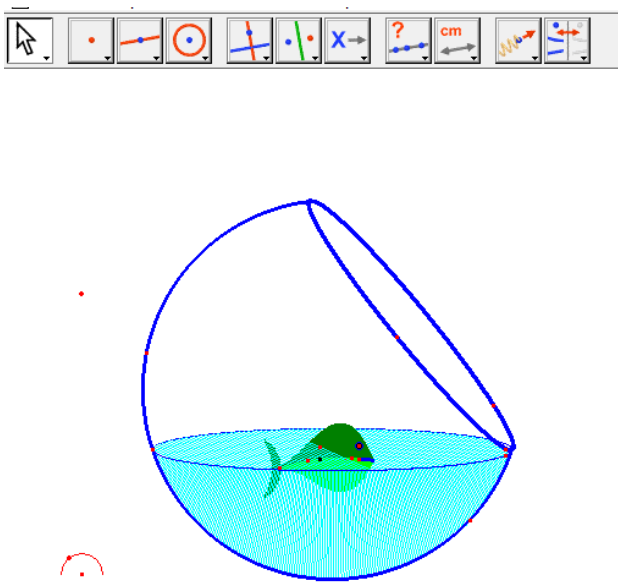

Figura 1. Una simulación tridimensional del vaciado de una pecera, con Cabri.

Mientras en otras partes del país se desarrollaban proyectos de largo alcance sobre el uso de tecnología en el aula como el caso de EMAT (Rojano, 2003) y se elaboraban tesis de posgrado sobre el tema, en la Universidad de Sonora (Unison) nos estábamos planteando la posibilidad de incorporar la tecnología en algunos temas de los cursos de matemáticas.

\subsection{Las versiones gráfico dinámicas de algunos temas.}

\subsubsection{Números Complejos: una presentación gráfica}

En algunos de estos trabajos, ya se contaba con versiones más orientadas hacia las representaciones gráficas, éste fue el caso del Módulo sobre números complejos (Soto, 2002), que se había escrito para el curso universitario de Álgebra, como una alternativa al acercamiento casi estrictamente algebraico que se usaba en estos cursos. Las ideas principales para escribir este módulo habían sido tomadas de (Markushévich, 1984), se incluye una discusión sobre las operaciones con números reales representados como "flechas" cuyos argumentos pueden ser de $0^{\circ} \mathrm{o} 180^{\circ}$, una discusión sobre la existencia del número $i$ como la necesidad de resolver la ecuación $x^{2}+1=0$, la representación de números complejos en el plano, las operaciones de suma y multiplicación y el cálculo de potencias y raíces de un número complejo. Todos los temas inician con una discusión gráfica asociada a la representación de números complejos como "flechas" en el plano, para pasar gradualmente a las representaciones algebraicas y establecer relaciones entre los elementos de ambas. Es así como el material se centra en proveer de situaciones en las que se pueden visualizar las propiedades algebraicas de los números reales y complejos, intentando hacer visible el origen de las propiedades de estos últimos.

Con una aproximación como la descrita, resultó natural la actualización del módulo usando Cabri para convertir las gráficas estáticas en dinámicas, simplemente introduciendo applets como el que se muestra en la Figura 2, con los que se planteaban actividades complementarias a los temas. El applet corresponde a las operaciones de suma y resta de complejos. La construcción de estos applets utilizaba las herramientas predefinidas en Cabri de vectores y puntos de coordenadas reales para representar los números complejos; agregando textos dinámicos que permitieran observar los valores numéricos de los números complejos representados. El módulo se sigue usando como material de enseñanza en la Unison, aunque algunos profesores han reconstruido los applets en GeoGebra. 


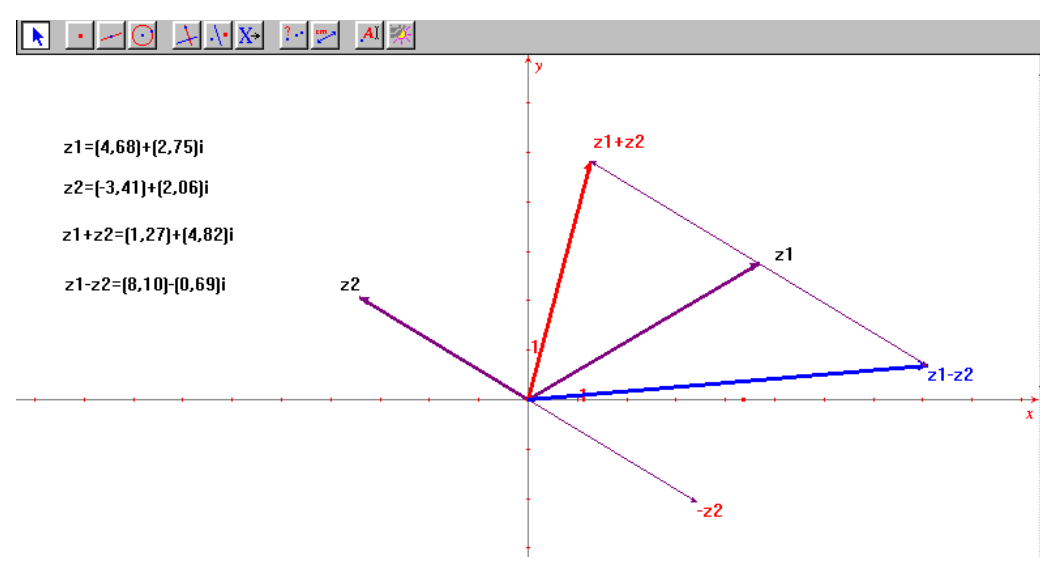

Figura 2. Suma y resta de números complejos, representadas con Cabri.

Se destaca que en esta etapa el material fue diseñado con base en la exploración de gráficas estáticas y la familiarización con el software permitió su extensión al uso de Cabri en el aula para su exploración con gráficas dinámicas. Así mismo, las construcciones eran suficientemente simples como para proponer que los estudiantes las replicaran en el aula como parte de la exploración gráfica. En el siguiente ejemplo, se parte de la experiencia con los temas elementales de números complejos para proponer el estudio de algunas funciones especiales en el curso de Variable Compleja.

\subsubsection{Una exploración gráfica de las propiedades de las transformaciones de Möbius}

Tras la experiencia en la representación y manipulación de números complejos en el plano, se propuso el estudio de un tipo de transformaciones conformes, dada su importancia en la resolución de problemas de la física y la matemática misma. Las transformaciones estudiadas se caracterizan por ser fraccionales lineales, de la forma $T(z)=\frac{a z+b}{c z+d}$, donde $a, b, c$ y $d$ son números complejos tales que $a d-b c \neq 0$.

Se eligieron las transformaciones de Möbius por sus propiedades geométricas principales:

i. Transforman círculos en círculos, incluyendo el caso de las rectas como circunferencias en el plano complejo extendido $\mathbb{C}_{\infty}$.

ii. Preservan ángulos, es decir, si dos curvas se intersecan en un ángulo $\alpha$, sus imágenes se intersecan también formando un ángulo $\alpha$.

Al representar $\mathbb{C}_{\infty}$ con el plano de Cabri y a los números complejos como vectores, se facilita la visualización de las propiedades descritas arriba, las cuales comúnmente son presentadas de forma verbal o con sólo un par de ejemplos estáticos. La posibilidad de manipular un número complejo restringido a una circunferencia cualquiera y generar así su imagen en el plano, permitió a los estudiantes observar la universalidad de esta propiedad y por lo tanto su trascendencia. 


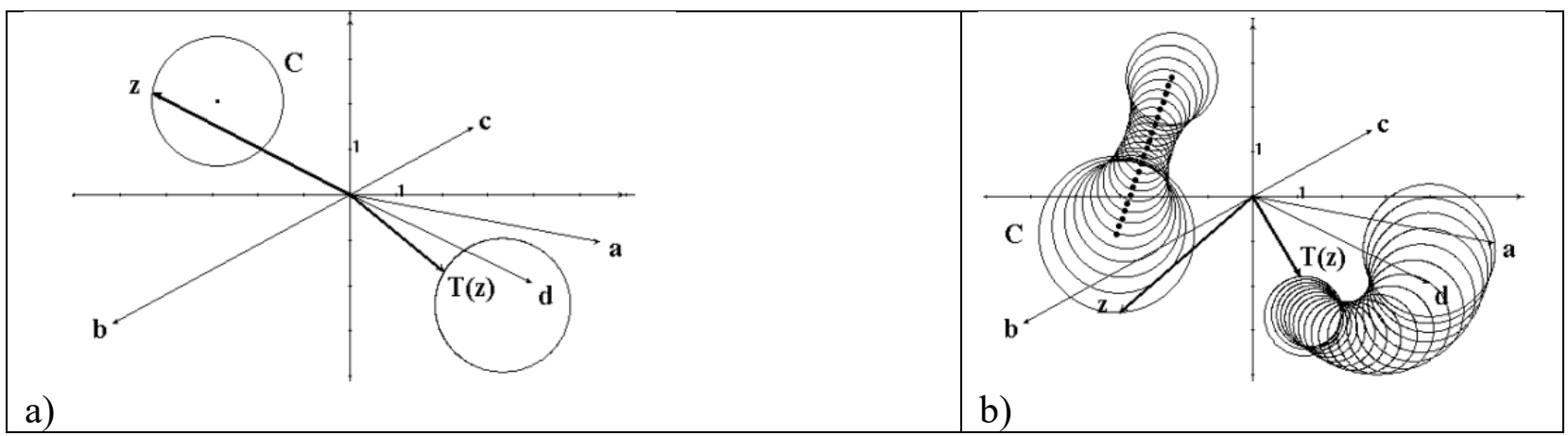

Figura 3. La imagen de una circunferencia y de una familia de circunferencias, bajo una transformación de Möbius.

En el apartado técnico, el desarrollo de las construcciones en Cabri requirió la implementación de las operaciones de suma, producto y cociente desarrolladas para el tema de complejos. Adicionalmente, se utilizaron vectores con extremos dentro de circunferencias para el complejo $z$ (argumento de la transformación) y el uso del rastro con la herramienta Traza y el lugar geométrico generado en automático para resaltar la imagen de la circunferencia. Estos últimos elementos técnicos son los que permiten plantear a los estudiantes la observación de la generalidad de las propiedades geométricas, permitiendo primero el arrastre de $z$ sobre la circunferencia (Figura 3a) y posteriormente el arrastre de la circunferencia misma para observar que la transformación conserva cualquier circunferencia (Figura 3b).

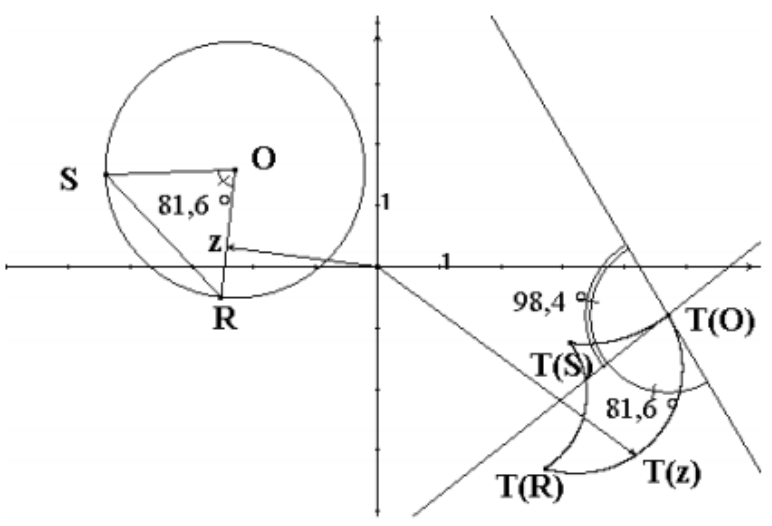

Figura 4. Una exploración sobre la conformidad de las transformaciones de Möbius.

El método utilizado para construir una transformación de Möbius permite también plantear a los estudiantes la manipulación de los parámetros $a, b, c, \mathrm{y} d$, ya que cada uno es un vector en la pantalla. De tal manera, se explora también el efecto gráfico que tiene cada parámetro en la manera de conservar las circunferencias. Con las exploraciones planteadas, se espera que los estudiantes puedan predecir el efecto de una transformación, así como deducir las preimágenes de algunos casos particulares de circunferencias. La conservación de ángulos es estudiada en un ambiente de Cabri adicional, interpretados como sectores circulares, de los cuáles se puede conocer su imagen fácilmente aplicando los resultados de la sección anterior. Para medir los ángulos, se agregan tangentes a la construcción y se utiliza la herramienta de medición de Cabri (Figura 4).

Destacamos que, en esta propuesta las tareas planteadas son desde un inicio exploraciones dinámicas, incluyendo la construcción de varios elementos por parte de los estudiantes. Así mismo, que la propuesta de enseñanza parte de la construcción y observación de propiedades, explorando la extensión de éstas incluso 
en casos extremos como en el de las rectas como circunferencias que pasan por el punto al infinito. Se pretende aquí, que las exploraciones permitan a los estudiantes resolver problemas planteados en Cabri y también problemas tradicionales con lápiz y papel.

\subsubsection{Un método gráfico para graficar un polinomio.}

Este trabajo tiene una historia extraña. En el año de 1999, buscando las presentaciones que se hacían del tema de polinomios en libros de texto en desuso, en un viejo texto de Teoría de Ecuaciones (Turnbull, 1947 pp. 27-30) encontramos un método para evaluar polinomios en un número real arbitrario. El método resultó sorprendente, era gráfico y había sido diseñado por un Eduard Lill, matemático de quien no se había oído hablar en la región. En el texto mencionado lo recomendaban para aquellos casos en los que los coeficientes del polinomio eran decimales difíciles de manejar, pero solo se requiere una solución aproximada. El hallazgo nos entusiasmó porque si se podía traducir el método a Cabri, podría obtenerse a partir de un segmento de medida $x_{0}$, otro segmento de medida $p\left(x_{0}\right)$ lo cual permitiría construir un polinomio dinámico en pantalla, con los coeficientes directamente manipulables, simplemente arrastrando puntos en la construcción. Este logro pareciera trivial por las facilidades del software moderno, pero en Cabri no había manera directa de construir funciones, ni siquiera polinomios.

El problema técnico de la construcción resultó sencillo, se le dedicó más tiempo a conseguir las referencias sobre el método y su autor, porque en aquel tiempo se sabía realmente poco sobre ello. Se tuvo que escribir al foro Historia Matemática, actualmente fuera de funcionamiento, pero cuyos mensajes se conservan en la una página internet (González, 2000). Ahí nos informaron que existían dos artículos en los que Lill (1867, 1868) exponía su método. Aunque ahora estos artículos están en línea en varios sitios, los tuvimos que mandar pedir a Francia, porque la revista en la que se escribieron dejó de publicarse en 1927. En Soto (2002b) se ofrece una explicación del método, una demostración de que funciona para el caso particular de un polinomio de grado tres y las instrucciones detalladas de cómo construir la versión dinámica del método, con Cabri. En la construcción final (Figura 5) del trabajo se grafica el polinomio $p(x)=x^{5}+4 x^{4}+2 x^{3}-$ $4 x^{2}-x+1$

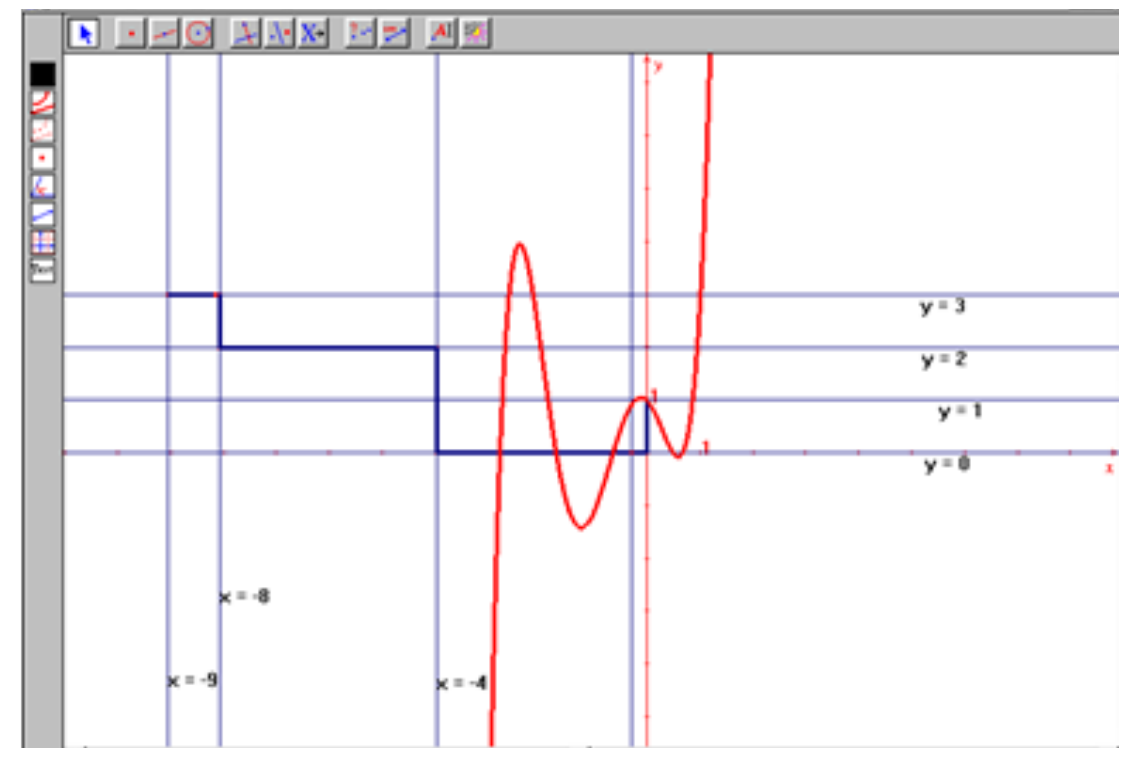

Figura 5. Gráfica dinámica de un polinomio de grado 5, construido con Cabri y usando el método de Lill. 
Este trabajo tuvo dos finales, uno bueno y uno malo. El bueno fue que el trabajo fue premiado por la Sociedad Matemática Mexicana (SMM) en el concurso "Matemáticas Aplicadas y su Enseñanza", en el año 2002 y la mala es que la SMM se quedó con los derechos de autor para publicarlo y nunca lo publicó.

Aunque el trabajo no fue publicado, excepto un resumen en las memorias del PME-NA XXII (Fernández, 2000, p. 268), el potencial de la construcción para representar polinomios dinámicos de manera gráfica y algebraica simultáneamente, en una época en la que todavía no existía software alguno que hiciera eso, fue aprovechada para diseñar otro fascículo para el curso universitario de Álgebra Superior, titulado: "Polinomios y raíces: una presentación gráfica", pero de ese trabajo se hablará en el siguiente apartado.

\subsubsection{Material Didáctico: Polinomios y raíces: una presentación gráfica}

Siguiendo con el enfoque definido en el material sobre Transformaciones de Möbius, se plantea ahora el estudio de polinomios reales y sus raíces. Para ello, se presentan construcciones predefinidas en las que los estudiantes no necesitan conocer Cabri, ya que es suficiente la manipulación directa de parámetros algebraicos para la exploración de las construcciones gráficas y el establecimiento de relaciones entre ambas. En un segundo momento, por limitaciones técnicas del software dinámico se requiere el apoyo de software de representaciones estáticas para explorar algunas de las propiedades. Conforme a las limitaciones de la época, era necesario prevenir a los posibles usuarios sobre la necesidad de equipo de cómputo para los estudiantes, requiriendo comúnmente el uso de un laboratorio de cómputo.

Como se mencionó arriba, el método de Lill permite la representación gráfica de polinomios como el lugar geométrico de un punto que se obtiene a partir de las reflexiones sobre una poligonal. En esta propuesta, se oculta todo el procedimiento geométrico necesario para mostrar la gráfica del polinomio y se agregan segmentos cuyas longitudes representan a cada coeficiente del polinomio (de grado fijo); el estudiante no tendrá que reconstruir el método de Lill, sólo interactuar con los segmentos. Posteriormente (Figura 6), se presenta una construcción similar en la que se agrega la gráfica de la derivada del polinomio, con lo que se pueden explorar las relaciones entre las raíces de $p(x)$ y $p^{\prime}(x)$, se estudian aquí los casos de raíces nulas $(r=0)$ y raíces múltiples.

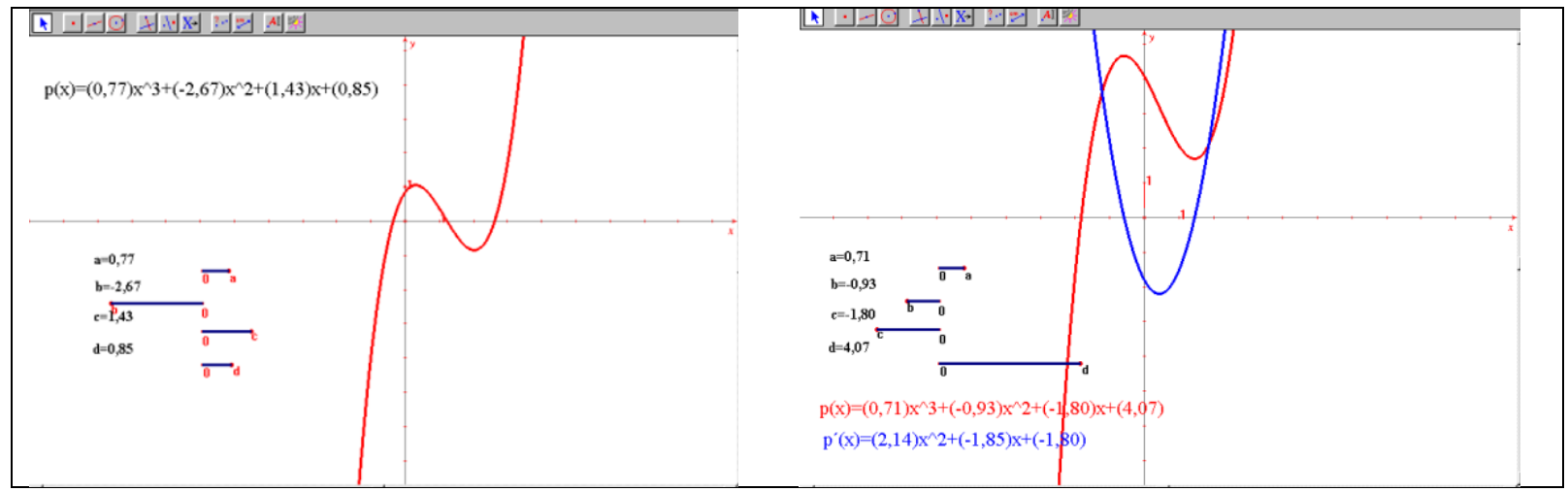

Figura 6. Comparación entre un polinomio de grado 3 y su derivada.

Para finalizar, se plantea el desarrollo de un método para aproximar las raíces reales y complejas de un polinomio, partiendo de una de las ideas geométricas en la demostración de Gauss para el Teorema Fundamental del Álgebra (Coxeter, 1977). El método parte de la separación de la parte compleja y la parte real del polinomio, evaluando $p(x+i y)$, desarrollando las operaciones y separando la ecuación en números complejos $p(z)=0$ en el par de ecuaciones reales $q_{1}(x)=0$ y $q_{2}(y)=0$, basados en que la igualdad de un número complejo a cero implica que tanto la parte real como la parte imaginaria deben ser nulas. 
Mientras que este método se puede aplicar de forma completamente algebraica, se plantea la exploración de cada paso en Maple V (ver Figura 7), para visualizar las características de las raíces reales y complejas. Es notable que el trabajo en Maple difiere bastante del trabajo en Cabri, ya que el primero está orientado a la resolución de problemas en matemáticas avanzadas, mientras que Cabri estaba orientado a la exploración gráfica y a las construcciones geométricas. De tal manera, la interacción con el software es ahora por medio de comandos y se pierde la manipulación directa de las gráficas, teniendo que dedicar un tiempo considerable a la familiarización con los comandos algebraicos de Maple.
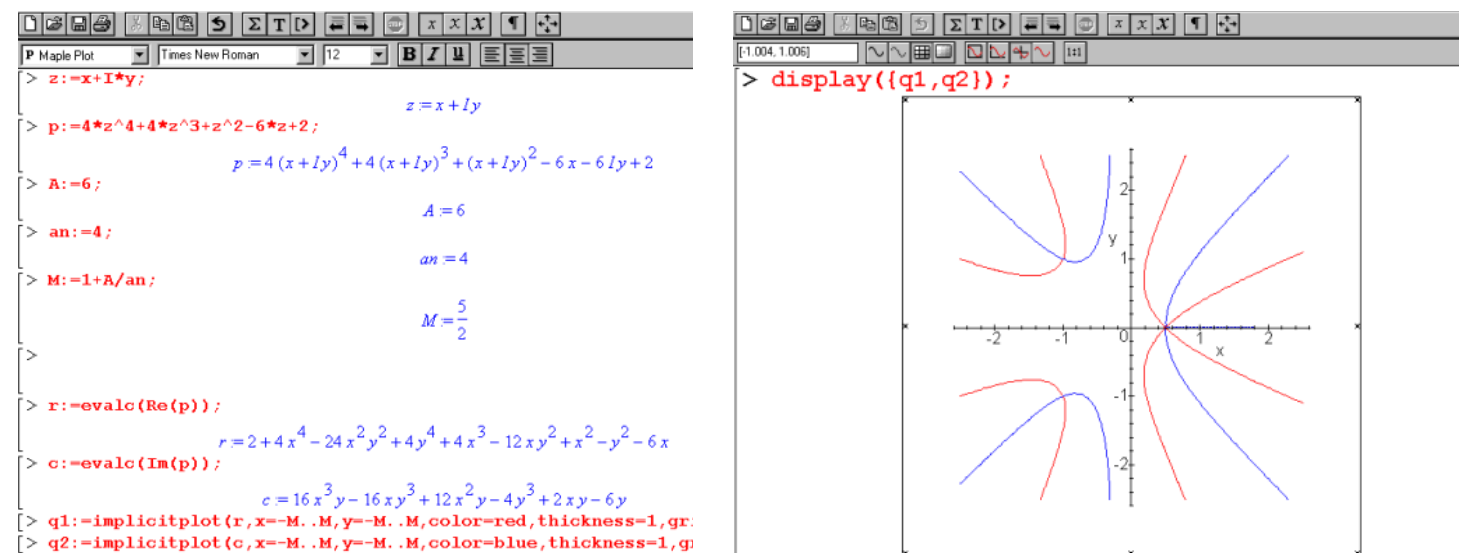

Figura 7. La representación gráfica de las raices reales y complejas exploradas con Maple.

La visualización de polinomios en el plano complejo permite ahora el planteamiento de problemas más generales que en las etapas anteriores, planteando a los alumnos la búsqueda de todas las raíces de polinomios de grado alto (mayor que cinco). El software permite a los estudiantes graficar los polinomios propuestos, se les plantea intentar predecir la cantidad de raíces utilizando el criterio de los signos y después que comprueben sus predicciones mostrando las gráficas de la parte real y parte imaginaria de los polinomios, asociando las raíces de $p(x)$ a las intersecciones de $q_{1}(x)$ y $q_{2}(y)$.

Es destacable que en esta propuesta continúa siendo el enfoque de exploración gráfica el que guía el diseño y la suficiente familiaridad con el software lo que hace posible la construcción de los ambientes prediseñados para los estudiantes, permitiendo cambiar el apoyo digital por otro, cuando no es posible generar las representaciones o manipulaciones deseadas. Las actividades propuestas incluyen ahora, la exploración gráfica, la manipulación directa e indirecta de las gráficas y finalmente el cálculo algebraico simbólico para la comprobación de resultados previstos. Por otro lado, la poca experiencia con el uso del software por parte de los estudiantes sigue influyendo en el tipo de tareas e interacciones que se plantean, limitándose a la manipulación de dos ambientes dinámicos prediseñados y, en el caso de Maple, al uso de programación con comandos específicos.

\subsubsection{A propósito de un instrumento que grafica cónicas}

A pesar de que estábamos convencidos del potencial didáctico de los Sistemas de Geometría Dinámica (conocidos como DGS por sus siglas en inglés) para el año 2000 seguíamos buscando formas sistemáticas de incorporar esta tecnología digital al trabajo docente. En esa búsqueda, nos encontramos un artículo de Santos (2000), en el cual reportaba el ejemplo que muestra la Figura 8. Se trata de una construcción en Cabri que fue presentada a los estudiantes de bachillerato y que consiste en un punto $P$ sobre la recta $k$, un punto fijo $C$, un punto $Q$ sobre la una circunferencia centrada en $C$, el punto $R$ simétrico a $C$ con respecto a $k$ y el punto $S$ que se obtiene al intersecar la recta $P Q$ con la recta $R C$. El lugar geométrico del punto $S$, cuando el 
punto $Q$ se arrastra sobre la circunferencia, es una cónica. Y haciendo variar $P$ sobre la recta $k$, pueden obtenerse diferentes cónicas. El aparato virtual construido de esta manera resulta sorprendente y contrasta con la sencillez de su construcción en Cabri (ver Figura 8). En el artículo se reportaba que los estudiantes podían identificar las diferentes cónicas y algunos de sus elementos. El artículo era interesante e ilustraba el uso que podría darse a los applets construidos con Cabri, en el salón de clase. Pero a nosotros nos llamó la atención otra cosa, ¿cuáles eran las razones matemáticas por las cuales el punto $S$ trazaba cónicas?

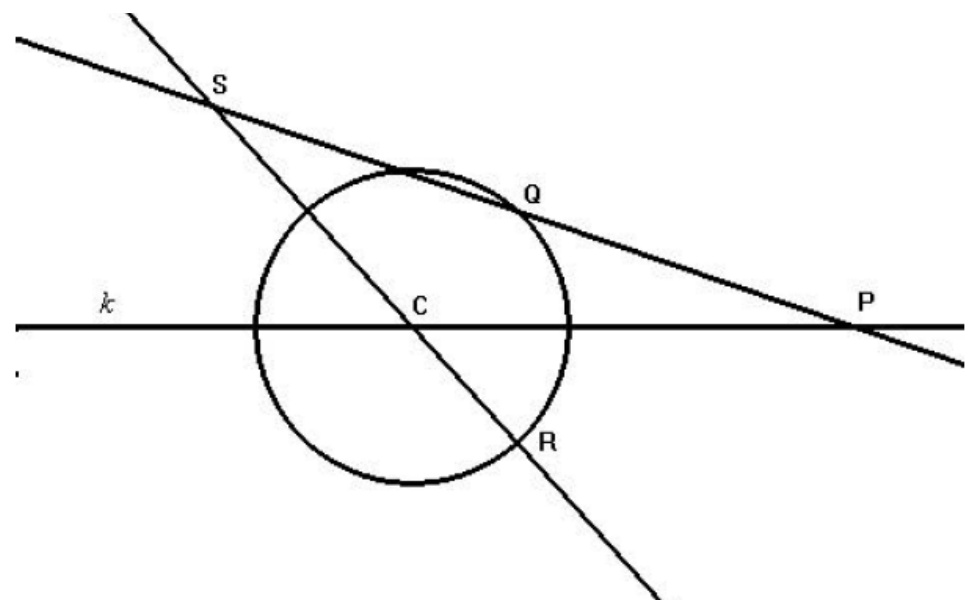

Figura 8. La construcción de un conicógrafo dinámico con Cabri

Para dar respuesta a esta pregunta, tomamos la definición general de cónica Lehmann (1972, p. 220):

"Definición. Dada una recta fija l y un punto fijo $F$ no contenido en esa recta, se llama cónica al lugar geométrico de un punto $S$ que se mueve en el plano de ly $F$ de tal manera que la razón de su distancia de F a su distancia de l es siempre una constante positiva. La recta fija l se llama directriz, el punto fijo $F$, foco, y la constante positiva, a la que designaremos por e, excentricidad de la cónica."

Aunque la definición aparece en un texto de Geometría Analítica, no exige el uso de las herramientas de esta Geometría y por ello decidimos ofrecer una prueba con las herramientas de la Geometría Sintética. La demostración de que los lugares geométricos trazados por el punto $S$ son cónicas, no resultó un problema difícil, la dificultad principal estriba en proponer una recta $l$ como directriz y un punto $F$ como foco y aquí es en donde el software resultó imprescindible para conjeturar la localización de la recta $l$ y el punto $F$ (ver Figura 9). Por eso insistimos en el trabajo, que hemos usado el software para conjeturar sobre la existencia y ubicación de $l$ y $F$, usando además el método de análisis para suponer que el problema de la posición de $l$ y $F$ estaba resuelto. La directriz $l$ resultó coincidir con la mediatriz del segmento $C P$ y el foco $F$ al que alude la definición resultó coincidir con el punto $C$. 


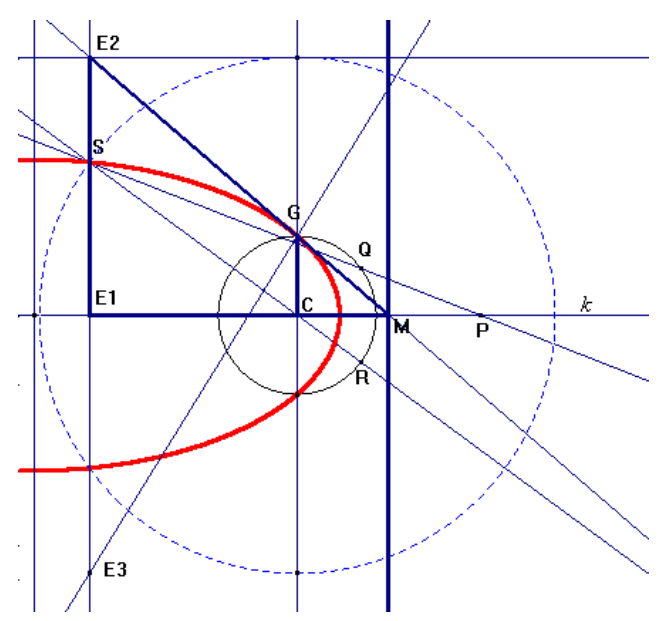

Figura 9 Exploración con Cabri para localizar la directriz y el punto $F=C$.

Encontrada una explicación matemática sobre la naturaleza de las curvas trazadas por lo que en el trabajo llamamos conicógrafo, surgió otra pregunta derivada también de la exploración con Cabri. Al explorar con detalle la manera como funcionaba el conicógrafo, encontramos varios elementos para pensar que el aparato funcionaba, porque podría tratarse de un conicógrafo espacial en donde el círculo con centro en $C$, representaba un corte sobre un cono y las cónicas generadas podrían representar los cortes al cono por un plano que dependería de $P$; es decir que podría tratarse de un aparato espacial que solo estaba mostrando la "vista aérea" (ver Figura 10).
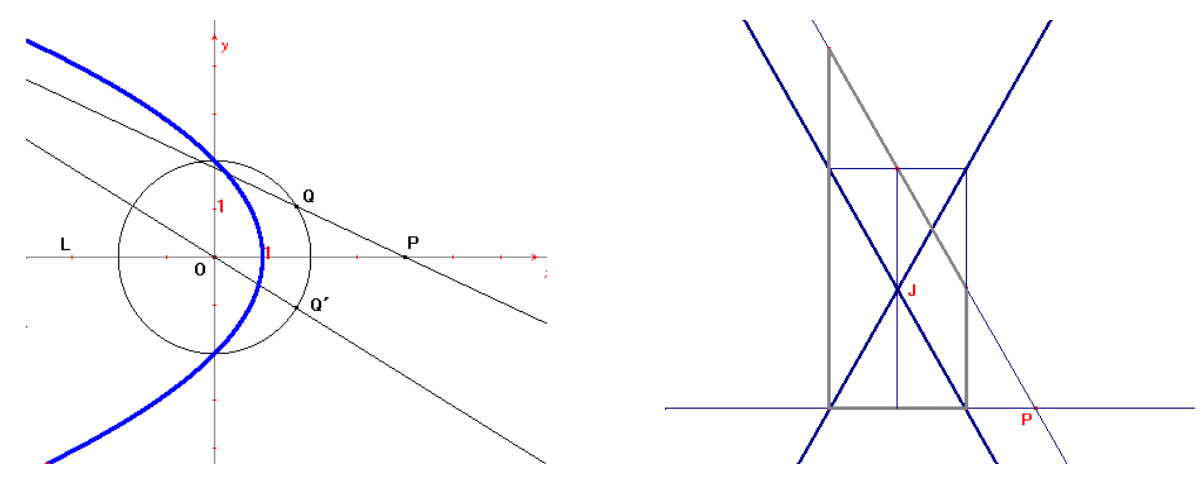

Figura 10. Una comparación entre el conicógrafo plano y el posible conicógrafo espacial.

De nueva cuenta, Cabri resultó imprescindible para conjeturar la posición del cono que supusimos y para demostrar sin métodos analíticos que efectivamente el conicógrafo tiene una versión tridimensional, con la que puede conectarse el concepto de cónica con los desarrollos de Apolonio de Perga. En virtud de que Cabri no contaba en esa época con una vista tridimensional, tuvimos que simular la versión tridimensional del conicógrafo, que al final quedó como muestra la Figura 11. 


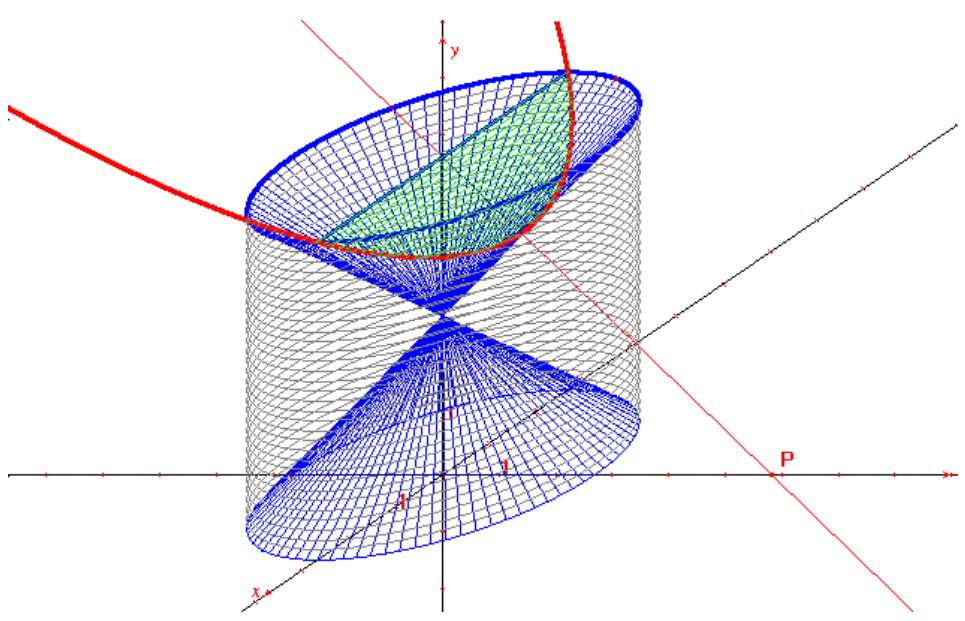

Figura 11. La interpretación espacial del conicógrafo, construida con Cabri.

El artículo en donde se describen los detalles de este trabajo (Soto, 2002), fue publicado en la revista del Departamento de Matemáticas de la Unison.

La principal enseñanza que nos ha dejado este trabajo es que el uso de Cabri para explorar y resolver problemas, es una herramienta que hace posible aprender matemáticas explorando, conjeturando y generando ideas sobre la solución de los problemas. Algunas reflexiones sobre este trabajo se presentaron en el IV Congreso IberoCabri que se llevó a cabo en Córdoba, Argentina en 2008. Fue el último evento sobre Cabri al que asistimos y aunque una de nuestras tesistas presentó los avances de su trabajo dos años después en el V Congreso de IberoCabri, era evidente que los tiempos de auge de este software ya habían pasado y que muchos de los que alguna vez lo utilizamos, estábamos emigrando a GeoGebra, que no solo es más potente y versátil, sino que además es gratuito.

\section{La fundamentación didáctica para usar DGS}

Todos los trabajos desarrollados hasta aquí provenían de la preocupación por mejorar nuestros materiales de enseñanza sin más fundamento que la intuición y el empirismo, siguiendo inicialmente los criterios o exploraciones gráficas conocidas para algunos temas avanzados. En todos los casos, se planteaba actividad para los estudiantes esperando que fuera de utilidad, principalmente por alejarse de lo tradicional y por favorecer una introducción intuitiva a temas abstractos. En la siguiente etapa, un acercamiento a la investigación en Matemática Educativa permitió el inicio de una fundamentación teórica para la actividad de diseño de actividades didácticas.

\subsection{Sobre dificultades de aprendizaje en ambientes tecnológicos}

Las experiencias empíricas descritas arriba sobre el diseño de materiales didácticos y el uso de software dinámico en cursos de matemáticas permitieron adentrarse en lo que la literatura especializada describía como problemas y dificultades de aprendizaje. Entre los distintos enfoques teóricos disponibles en esta época, se observó afinidad particularmente con el enfoque de visualización y de registros de representación semiótica de Duval (1999). Desde este enfoque teórico, se había argumentado ya la importancia de la conversión entre distintos registros, generando dificultades de aprendizaje en particular para el Álgebra Lineal (Pavlopoulou, 1993). 
De manera conforme al tipo de investigación en Matemática Educativa de la época, la experiencia en las exploraciones gráficas con Cabri en temas que pueden ser interpretados vectorialmente, llevó a una investigación doctoral sobre Las dificultades para la conversión gráfico-algebraica relacionadas con conceptos básicos de la teoría de espacios vectoriales en $\mathbb{R}^{2}$ y $\mathbb{R}^{3}$ (Soto, 2003). Siguiendo resultados de estudios exploratorios sobre el uso de software dinámico para atender dificultades de aprendizaje (Sierpinska, Dreyfus \& Hillel, 1999), se plantea la posibilidad de que las dificultades detectadas puedan ser contrarrestadas en alguna medida y de verificar a la vez si el ambiente tecnológico propuesto no crea nuevas dificultades. De tal manera, se evaluó el desempeño de los estudiantes al resolver las diversas actividades sobre temas como: elementos neutros, elementos inversos, multiplicación por escalar, dependencia e independencia lineal, conjuntos y espacios generados, combinaciones lineales, transformaciones lineales y valores y vectores propios.

El marco teórico de Duval permitió analizar más detalladamente el desempeño de los estudiantes, identificando características de algunas representaciones que pueden facilitar u obstaculizar la construcción de significados, como se observa con el caso del vector cero (Soto, 2003, pp. 190-191). La representación gráfica del vector cero como la ausencia de una flecha se aprecia como un obstáculo para construir algunos de los significados pretendidos en las actividades, como la neutralidad aditiva " $u+0=u$ para todo vector $u$ ". Al parecer ésta y otras propiedades no fueron construidas a partir de las tareas propuestas en las actividades, debido a confusiones generadas por las restricciones del registro al representar el vector cero ( $\mathrm{p}$. 188).

En lo que respecta a las representaciones dinámicas, se observó que las ventajas del software vienen acompañadas de sus propias complicaciones. Como ya se había reportado en situaciones similares (Sierpinska et al., 1999), la representación gráfica de vectores arbitrarios o de cualquier vector del plano se mostró conflictiva en la propuesta de enseñanza. Se esperaba que las herramientas del software permitieran interpretar flechas que se podían arrastrar por el plano como equivalentes a la letra " $v$ " en la expresión " $v \in$ $\mathbb{R}$ ", sin embargo, en varias ocasiones se interpretaron tales flechas como un vector específico el cual podía cambiar, o como una flecha que se estira.

En general, se pudo observar una situación que se manifestó globalmente en la época: aunque el software dinámico permite plantear y realizar exploraciones gráficas de temas matemáticos abstractos y avanzados, su sola inclusión no elimina las dificultades de aprendizaje, más aún, suele generar nuevas dificultades. De tal manera, se regresó a la actividad de diseño de actividades didácticas, pero ahora con el panorama teórico y un énfasis en la atención a las dificultades de aprendizaje detalladas por resultados de investigación.

\subsubsection{Propuesta de enseñanza basada en la conversión Gráfico-Algebraica, con apoyo de}

\section{GeoGebra}

Como una ramificación de algunos de los resultados y experiencias de enseñanza en álgebra lineal, se desarrolló una propuesta de enseñanza que permitiera construir un significado gráfico para el concepto de transformación lineal (Romero, 2010) para saber más sobre los efectos en el aprendizaje de los estudiantes, si el concepto se introduce a partir de representaciones gráficas. Para favorecer la construcción de un significado de las transformaciones lineales, y con la intención de reducir dificultades de aprendizaje, se diseñó una secuencia de actividades que se apoya en ambientes preconstruidos que utilizan representaciones dinámicas. Con este proyecto entramos a nueva etapa, en la que se enfocaron esfuerzos al diseño de actividades estructuradas y con fines específicos de aprendizaje, basados en los resultados de la investigación 
sobre dificultades de aprendizaje. Con este proyecto se consolida además la transición a GeoGebra como base para el desarrollo de ambientes dinámicos,

Se refinaron algunos de los criterios empíricos para el diseño de actividades, ahora en términos del marco de registros de representación, planteando que:

1. El registro de representación en el que se inicia el estudio de algún objeto matemático afecta el nivel de comprensión que se puede llegar a obtener de él.

2. El registro gráfico permite la creación de un ambiente enriquecedor, en el que se pueden caracterizar las transformaciones lineales por sus propiedades gráficas.

3. Los ambientes dinámicos diseñados con GeoGebra pueden facilitar a los estudiantes la observación y comprobación de las propiedades gráficas de una transformación lineal mediante la manipulación directa en pantalla, facilitando con ello la conversión gráfico-algebraica.

Estos supuestos teóricos guiaron posteriormente la etapa de diseño de actividades, combinados con las herramientas novedosas de GeoGebra para representar y manipular vectores. Principalmente se aprovechó que en GeoGebra se pueden definir tanto objetos geométricos como algebraicos, y que se cuenta de inicio con prácticamente la misma colección de herramientas geométricas que en Cabri, pero agregando una variedad de objetos y manipulaciones algebraicas. De tal manera, se pudieron construir materiales para la manipulación de vectores en el plano, que permitieran la exploración de las transformaciones lineales, observando las propiedades de linealidad de forma gráfica, así como sus interacciones y consecuencias.

Las construcciones en GeoGebra aprovechan la potencia del software para representar de manera separada el plano del dominio de la función, del plano del contra-dominio y permiten al estudiante diversos tipos de manipulaciones directas sobre la pantalla de la computadora (ver Figura 12).

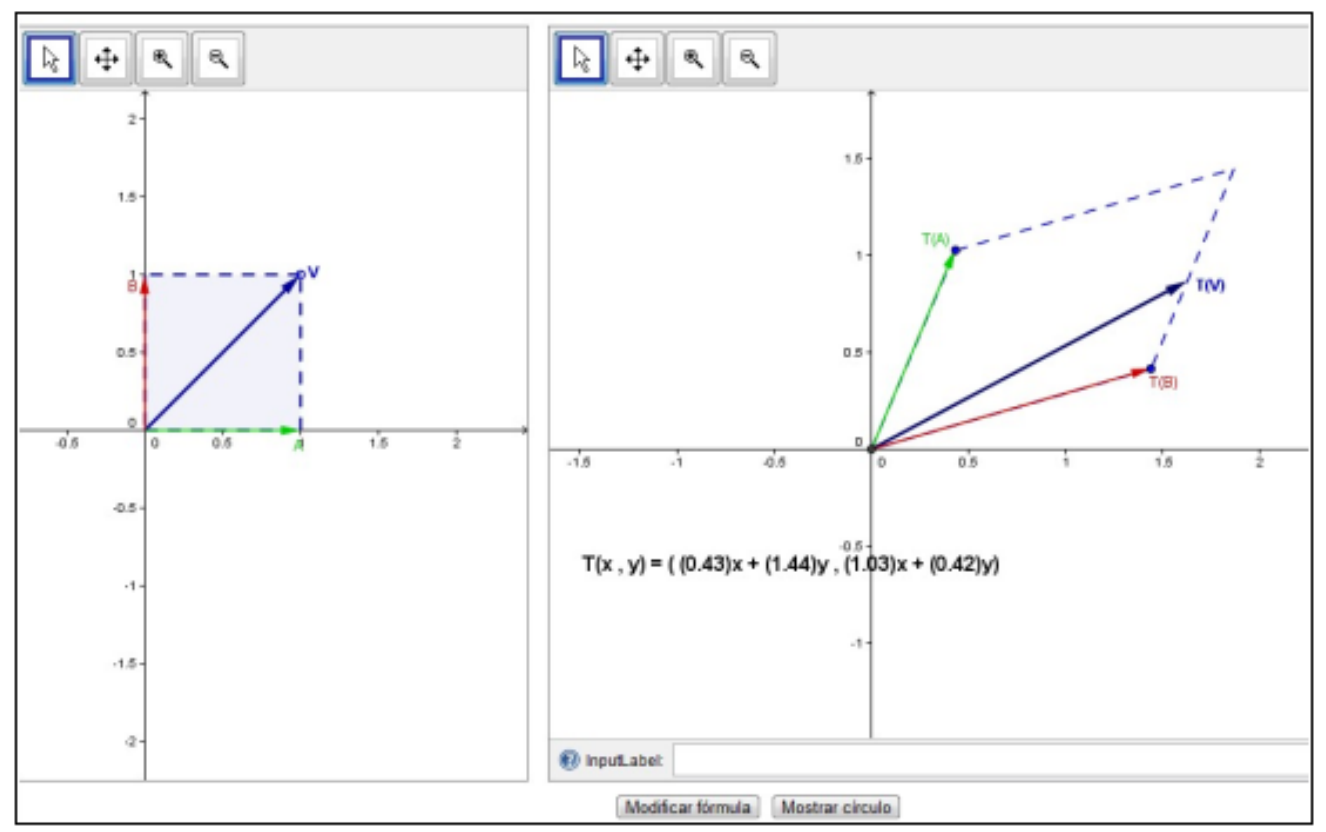

Figura 12. La discusión gráfica sobre las propiedades definitorias de una transformación lineal, en el ambiente de GeoGebra. 
Se continuó la estrategia de distribuir ambientes preconstruidos, o applets como ahora se les conoce, evitando distraer a los estudiantes con los elementos del uso del software que no parecieran de provecho para el aprendizaje matemático buscado. En esta etapa se siguieron resolviendo problemas o limitaciones técnicas del software para generar el tipo de interacción buscada. De acuerdo con los principios del marco de registros de representaciones semióticas, resulta fundamental la identificación de las unidades figurales como unidades de significado y la búsqueda de correspondencias entre unidades figurales en representaciones de diferentes registros. En la versión de GeoGebra 2, se tenían disponibles las articulaciones entre representaciones gráficas y algebraicas, lo que facilitó mucho el diseño de las exploraciones y tareas y en general, sin embargo, para plantear la separación del plano del dominio y del plano imagen de una transformación líneal fue necesario complementar el uso de GeoGebra con programación en javascript (ver Soto, Romero \& Ibarra, 2012); de manera similar a cuando se complementaba el uso de Cabri con el de Maple.

Otro elemento técnico que se introdujo en este proyecto fue la distribución de los materiales en línea, aprovechando de nuevo la compatibilidad entre GeoGebra y los lenguajes de la red (principalmente html y javascript).

El proyecto sobre el aprendizaje de transformaciones lineales continuó como una de varias ramificaciones de esta línea de trabajo, avanzando a la investigación sobre las construcciones mentales específicas que se pueden desarrollar con este tipo de propuestas, articulando ahora el enfoque de representaciones con el de abstracción reflexiva de la teoría APOE (Romero, 2016). Como una nueva iteración de los refinamientos de las propuestas de enseñanza, se planteó una propuesta de enseñanza más extensa, que combinara los logros parciales de todas las etapas anteriores: exploraciones gráficas intuitivas, applets preconstruidos distribuidos en línea, exploraciones guiadas para que los estudiantes desarrollaran construcciones simples y planteamiento de problemas dentro y fuera de los ambientes dinámicos, para evaluar los aprendizajes específicos buscados.

\subsection{Actividades didácticas dirigidas a profesores de matemáticas de secundaria diseñadas con la metodología ACODESA.}

Éste es el título del trabajo que presentó Rodríguez (2011), como tesis de maestría bajo nuestra dirección. El trabajo refleja un viraje en nuestras preocupaciones a la hora de usar tecnología digital para elaborar materiales de enseñanza.

Si bien es cierto, tanto en la tesis doctoral de Soto (2003) como en la tesis de Romero (2010) las actividades de enseñanza con tecnología digital contaban con el respaldo de la teoría de representaciones construida por Raymond Duval, también es cierto que ésta es una teoría muy general, que no profundiza en el diseño de actividades de enseñanza. Ambos trabajos descansaban entonces sobre el propósito de promover la articulación de representaciones gráficas y algebraicas, porque en la teoría se establece que ésta es una condición para la aprehensión de los objetos matemáticos, pero no se especifican las formas concretas de promover esta conversión.

La tesis de Rodríguez (2011) en cambio, está basada en una metodología para el diseño de actividades didácticas que había venido desarrollando Hitt (2009), esta metodología llamada ACODESA (cuyas siglas significan aprendizaje en colaboración, debate científico, y auto reflexión) prescribe una serie de lineamientos para el diseño de actividades, que incluyen el uso de tecnología y que resultó apropiada para diseñar actividades con las siguientes características: a) el planteamiento de una situación problemática como 
punto de partida, b) la integración de diversos conceptos, no necesariamente contemplados en el mismo eje temático, c) la priorización de la acción del estudiante como detonadora de los aprendizajes y d) la incorporación de nuevas tecnologías en su instrumentación y e) que apoyen las competencias que la reforma educativa pretendía promover en los estudiantes. Específicamente la tesis utiliza el software GeoGebra.

ACODESA recomienda el uso previo de materiales didácticos manipulables, antes de usar tecnología y distingue usos diferentes de la tecnología, dependiendo de la etapa del diseño en el que se inserten. En las cuatro actividades didácticas incluidas en esta tesis, nos ha quedado clara la importancia de empatar el nivel de interacción estudiante-software, con los propósitos didácticos del diseño. En algún momento el estudiante tendrá que construir con GeoGebra, mientras que en otros solamente manipula objetos en applets preconstruidos.

\subsubsection{Libros de texto para el Colegio de Bachilleres de Sonora}

En el año 2014 nos involucramos en la escritura de los libros de texto para el Colegio de Bachilleres de Sonora. El proyecto de escribir los seis libros de texto de Matemáticas resultaba ambicioso, pero contábamos con la experiencia que algunos colegas teníamos en el diseño de actividades didácticas desarrolladas junto con nuestros tesistas o bien en los cursos de formación de profesores que habíamos ofrecido en los últimos años. En la escritura de los textos participamos 15 profesores del Departamento de Matemáticas de la Unison.

Los seis textos quedaron integrados por secuencias didácticas, cada una de las cuales se desarrolla en tres etapas: apertura, desarrollo y cierre, y la mayor parte de las secuencias iban acompañadas de applets construidos en GeoGebra. En el diseño se tomaron en cuenta algunos planteamientos de ACODESA, pero la metodología no se tomó al pie de la letra. Aprendimos durante la elaboración, que los applets construidos con GeoGebra tenían características muy diferentes cuando se hacían para las diferentes etapas de las secuencias. En la etapa de apertura, los applets tendrían que orientarse a la comprensión de la situación planteada, en el desarrollo a la resolución de la situación y en el cierre hacia la integración y formalización de los conceptos matemáticos que han emergido durante las dos etapas anteriores.

\subsection{Una propuesta metodológica para el diseño de secuencias didácticas para la matemática del nivel secundaria en un contexto tecnológico, utilizando GeoGebra}

El título de esta sección es el mismo que lleva la tesis de maestría presentada por Alvarado (2019) y en la que se ha aplicado una metodología de diseño que hemos venido construyendo y experimentando localmente, producto de nuestras experiencias de diseño y de nuestras reflexiones sobre la sistematización del uso de la tecnología digital para la enseñanza. Esta tesis en particular propone esta metodología a docentes de matemáticas de nivel secundaria, el objetivo de dicha propuesta es brindarle al docente una herramienta para elaborar diseños de secuencias didácticas con el fin de llevarlos al aula de clase; estos diseños tienen la particularidad de incorporar tecnología digital para la elaboración de la secuencia y para su implementación. La metodología fue elaborada con base en la articulación de: la estructura didáctica de Díaz- Barriga (2013), el método de enseñanza ACODESA de Hitt y colaboradores (Hitt y Cortés, 2009; Hitt, Cortés y Saboya, 2017) los desarrollos curriculares de Taba (1962). La metodología se ha puesto a prueba con un grupo de 11 docentes en un curso-taller de 40 horas, los resultados obtenidos fueron tres secuencias didácticas elaboradas por tres equipos de docentes en un contexto tecnológico (usando GeoGebra), donde se percibe que es posible realizar diseños aplicando esta propuesta metodológica. 


\section{Trabajos en desarrollo y perspectivas.}

La línea de trabajo aquí esbozada sigue generando proyectos de intervención y de investigación en matemática educativa, con un enfoque muy particular sobre el uso de tecnología en el aula.

Por un lado, orientados hacia la enseñanza del Álgebra Lineal derivados de los resultados de los trabajos originales sobre esta rama de la matemática (Soto, 2003), de algunos resultados adicionales sobre vectores propios (Soto \& García, 2003) y de la enseñanza de transformaciones lineales (Romero, 2010, 2016), enfocándose ahora en el aprendizaje de temas más especializados como el de espacios propios y espacios invariantes (Antelo-Lopez \& Romero, 2020).

Por otro lado, orientados hacia el aprovechamiento de los principios geométricos intuitivos para el desarrollo de propuestas de enseñanza, en particular esta orientación se ha materializado en un proyecto sobre las dificultades para justificar proposiciones geométricas al resolver problemas de lugares geométricos con GeoGebra (Soto, Urrea \& Romero, 2020). Este proyecto de investigación actualmente incluye el desarrollo de tres tesis de maestría sobre propuestas de enseñanza de las cónicas como lugares geométricos, recurriendo al uso de mecanismos articulados físicos y digitales, recuperando algunos elementos de la Geometría Analítica de Descartes.

Actualmente estamos trabajando en el rediseño del curso de Geometría Analítica que se ofrece a las carreras de Ciencias e Ingeniería de la Universidad de Sonora. En el nuevo curso se incorpora el uso de GeoGebra como herramienta de exploración, pero hay un mayor involucramiento de los estudiantes en las construcciones dinámicas.

A manera de conclusión, diremos que en este artículo hemos trazado a grandes pasos el camino que hemos seguido para incorporar la tecnología digital en la enseñanza de la matemática. Esta experiencia nos ha mostrado que la incorporación empírica que puede hacerse desde nuestra práctica docente es apenas una condición necesaria para incursionar en este tema. Rebasar el empirismo exige la profundización en las teorías de lo didáctico, no solo para entender las dificultades que plantea en estudiantes y profesores la enseñanza con tecnología, sino además para tratar de entender las difícultades que entraña el diseño mismo de las secuencias de enseñanza, que tampoco puede encasillarse en una actividad estrictamente práctica.

\section{Referencias}

Alvarado, J., (2019). Una propuesta metodológica para el diseño de secuencias didácticas para la matemática del nivel secundaria en un contexto tecnológico, utilizando GeoGebra. Tesis de Maestría sin publicar.

Antelo-Lopez, I. \& Romero, C. F. (2020). Construcciones mentales sobre subespacio invariante desarrolladas en una propuesta de enseñanza desde la teoría APOE. En: Sacristán, A.I., Cortés-Zavala, J.C. \& Ruiz-Arias, P.M. (Eds.). Mathematics Education Across Cultures: Proceedings of the 42nd Meeting of the North American Chapter of the International Group for the Psychology of Mathematics Education, Mexico. Cinvestav / AMIUTEM / PME-NA.

Coxeter, H. S. M. (1977). Gauss as a geometer. Historia Mathematica, 4(4), 379-396.

Díaz-Barriga, Á. (2013). Secuencias de aprendizaje. ¿Un problema del enfoque de competencias o un reencuentro con perspectivas didácticas? Profesorado, Revista de Currículum y Formación del Profesorado, 17(3), 11-33. Disponible en: https://recyt.fecyt.es/index.php/docenteado/article/view/41685/23758.

González, J. (2000, julio 26). Re: [HM] Metodo grafico de Lill. [Foro en línea]. Disponible en http://archives.math.utk.edu/hypermail/historia/jul00/0141.html. 
Laborde, J.-M., and Bellemain, F. (1992). Cabri Geometry II computer software. LSD2-IMAG Grenoble and Texas Instruments.

Lill, M. E. (1867). Résolution graphique des équations numériques d'un degré quelconque une inconnue. Nouvelles Annales de Mathématiques, ser. 2, 6: 359-362.

Lill, M. E. (1868). Résolution graphique des e quartions algébriques qui ont déracinés imaginaires. Nouvelles Annales de Mathématiques, ser. 2, 7: 363-367.

Markushévich, A. I. (1984). Curvas maravillosas. Números complejos y representaciones conformes. Funciones maravillosas. ( $2^{\mathrm{a}}$ ed.). Moscú: MIR.

Hitt, F. y Cortés, C. (2009). Planificación de actividades en un curso-taller sobre la adquisición de competencias en la modelización matemática y uso de calculadora con posibilidades gráficas. Revista Digital Matemática, Educación e Internet, $\quad$ 10(1), 1-30. Disponible http://revistas.tec.ac.cr/index.php/matematica/article/view/1977

Hitt, F., Saboya, M., and Cortés, C. (2017). Task design in a paper and pencil and technological environment to promote inclusive learning: An example with polygonal numbers. En G. Aldon, F. Hitt, L. Bazzini, y U. Gellert (Eds.), Mathematics and Technology (pp. 13-30). Cham, Switzerland: Springer International Publishing. https://doi.org/10.1007/978-3-319-51380-5

Hohenwarter, M. (2002). GeoGebra-a software system for dynamic geometry and algebra in the plane. (Unpublished master's thesis), University of Salzburg, Austria.

Turnbull, H. (1947). Theory of Equations. Oliver and Boyd, Edinburgh; InterScience, New York

Polya, G. \& Latta, G. (1976). Variable compleja, Limusa, México.

Rodríguez, M-A. (2012). Actividades didácticas dirigidas a profesores de matemáticas de secundaria diseñadas con la metodología ACODESA. (Tesis de Maestría sin publicar). Universidad de Sonora. México.

Romero, C. F. (2010). Una Introducción Gráfica al Concepto de Transformación Lineal Usando GeoGebra. (Tesis de Maestría sin publicar). Universidad de Sonora, México.

Romero, C. F. (2016). Aprendizaje de Transformaciones Lineales Mediante la Coordinación de Representaciones Estáticas y Dinámicas. (Tesis de Doctorado sin publicar). Cinvestav. México.

Sierpinska, A., Dreyfus, T. \& Hillel, J. (1999). Evaluation of a Teaching Design in Linear Algebra: The Case of Linear Transformations, Recherches en Didactique des Mathématiques, 19(1), 7-41.

Soto, J. L. \& San Martín Sicre, O. (2000). Constructing Meaning For Polynomials: Exploring Duval's RcpresentationsConversions With Cabri Geomctrc II. En M. Fernández (Ed), Proceedings of the 22nd Meeting of the North American Chapter of the International Group for the Psychology of Mathematics Education.

Soto, J. L. (2002). Números Complejos: una presentación gráfica. Material didáctico No. 1. Departamento de Matemáticas. Universidad de Sonora. México

Soto, J-L. (2002b). A propósito de un aparato que grafica cónicas. Arenario 2(2), pp. 113-138.

Soto, J. L. (2003). Polinomios y raíces: una presentación gráfica. Material didáctico No. 1. Departamento de Matemáticas. Universidad de Sonora. México

Soto, J. L. (2003). Un estudio sobre las dificultades para la conversión gráfico-algebraica relacionadas con los conceptos básicos de la teoría de espacios vectoriales en $R^{2} y R^{3}$. (Tesis de Doctorado sin publicar). Cinvestav, México.

Soto, J. L. \& García, M. (2002). A graphical exploration of the concepts of eigenvalue and eigenvectors in $\mathbb{R} 2$ and $\mathbb{R} 3$. En I. Vakalis (Ed.) Proceedings of the Second International Conference on the Teaching of Mathematics at the undergraduate level. Creta, Greece, John Wiley \& Sons

Soto, J. L., Romero, C. F. \& Ibarra S. E. (2012). El concepto de transformación lineal: una aproximación basad en la conversión Gráfico-Algebraica, con apoyo de GeoGebra. En F. Hitt \& C. Cortés (Eds). Formation à la recherche en didactique des mathématiques (pp. 38-49). Quebec, Canada, Loze-Dion éditeu.

Soto, J. L., Urrea, M. A. \& Romero, C. F. (2020). Dificultades para justificar proposiciones geométricas al resolver problemas de lugares geométricos con GeoGebra. En: Sacristán, A.I., Cortés-Zavala, J.C. \& Ruiz-Arias, P.M. (Eds.). Mathematics Education Across Cultures: Proceedings of the 42nd Meeting of the North American Chapter of the International Group for the Psychology of Mathematics Education, Mexico. Cinvestav AMIUTEM / PME-NA. 
Taba, H. (1962). La elaboración del currículum. Buenos Aires: Troquel.

Cómo citar este artículo: Soto Munguía, J. L., \& Romero Félix, C. F. Un recorrido por nuestra experiencia en la inclusión de software dinámico en el diseño de materiales didácticos. SAHUARUS. REVISTA ELECTRÓNICA DE MATEMÁTICAS, (5) 1, pp. 127-159. 